\title{
Biocompatible Packagings for Fully Implantable Multi-Panel Devices for Remote Monitoring of Metabolism
}

\author{
C. Baj-Rossi, A. Cavallini, T. Rezzonico Jost, M. Proietti, F. Grassi, G. De Micheli, and S. Carrara* \\ Laboratory of Integrated Systems, Swiss Federal Institute of Technology (EPFL), Lausanne, Switzerland \\ T-cell development group, Institute for Research in Biomedicine - IRB, Bellinzona, Switzerland \\ * e-mail address: sandro.carrara@epfl.ch
}

\begin{abstract}
This paper compares three different biocompatible packaging covers suitable to support full implantation of multipanel sensors for remote monitoring of metabolism. The three covers have been designed, realized and implanted in mice for 30 days. ATP and neutrophil concentrations have been measured at the implant site after the device was explanted, to assess the level of biocompatibility of the device.
\end{abstract}

Keywords-Fully implantable biochip; packaging; polycarbonate membrane; parylene $C$; epoxy resin

\section{INTRODUCTION}

Highly integrated and complex systems are more and more required in advanced health care as well as in translational medicine. In particular, the continuous and simultaneous monitoring of several molecular metabolites can be realized with fully implantable multi-panel devices that integrate several heterogeneous systems to address different device functions [1]. However, several aspects need attention in this new area of systems integrations: coupling nano and biomaterials with CMOS electronics [2], security and privacy constrains [3], new designs in power [4] and data transmission [5], and biocompatibility issues [6].

To reduce the effects of the foreign body reaction, an external biocompatible packaging is needed to ensure a correct integration with the surrounding tissue once the device is implanted. Polyurethane, among many other polymers, has been extensively used as an outer membrane to act as a biocompatible interface with the surrounding host tissue.

In this paper, we show the realization of a packaging for an implantable device that consists of the integration of the sensing platform, the integrated circuits (ICs) and the powering antenna, for the continuous monitoring of drugs and metabolites in small animals. Potential sources of inflammation are attributed to the shapes and size of the device, and to the material used. Multi-walled carbon nanotubes (MWCNTs), used to enhance the sensing signal, are entrapped in a chitosan matrix to prevent toxicity due to their nano-particle nature. Parylene $\mathrm{C}$ was employed to prevent the leaking of potential hazardous substances and the corrosion of the electronics from the biological fluids. Two different membranes were used to cover the sensing part of the device and here we present and discuss three different solutions (Fig. 1) for the packaging.

\section{MATERIALS AND METHODS}

\section{A. Integrated device fabrication and assembly}

The microfabrication of the sensing platforms was realized at the EPFL Centre of Micro-nano Technology (CMI). Silicon wafers with $500 \mathrm{~nm}$ of native oxide were chosen as substrate. Chip metallization was realized by evaporation of $10 \mathrm{~nm}$ of Ti, followed by $100 \mathrm{~nm}$ of Pt. Metal passivation was made via atomic layer deposition of $\mathrm{Al}_{2} \mathrm{O}_{3}$, followed by dry etching with Argon Ion Milling. Details on the microfabrication can be found in [7].

Figure 1A shows the photograph of the first implantable device. The passive sensing platform measures $2.2 \times 15 \mathrm{~mm}$, and host five independent Pt working electrodes (WE) with common reference (RE) and counter (CE) electrodes [8].

Figure $1 \mathrm{~B}$ and $1 \mathrm{C}$ show the photographs of the second and third version of the implantable device, respectively. The platforms measure $12 \times 11 \mathrm{~mm}$, in order to fit the size of the coil $(12 \times 12 \mathrm{~mm})$ and the wire bonding. The platform hosts an array of four independent cells in the three-electrode configuration, WE, CE and RE, all made in Pt [9].

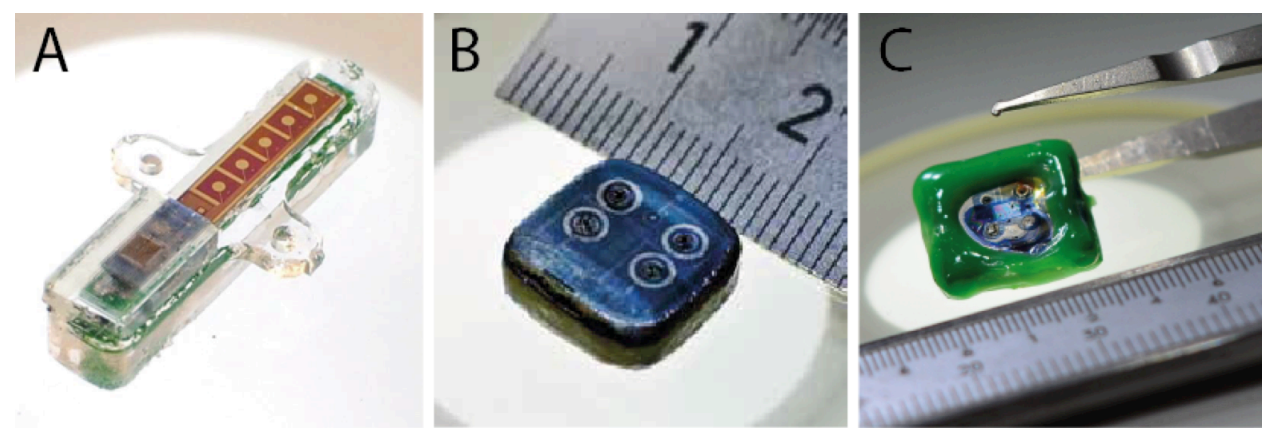

Fig. 1. Photograph of the three versions of the implantable device.

Swiss SNF project Sinergia CRSII2_147694 / 1, and i-IronIC project by Swiss Nano- Tera.ch. 


\section{B. Packagings}

For the realization of the first packaging for the electrode functionalization with MWCNTs, $0.1 \mu \mathrm{l}$ of a chitosanMWCNT suspension was then manually drop cast on the electrodes and dried in air. The surface of the biosensors was protected with a polycarbonate membrane (Cyclopore track etched membrane, cut off $100 \mathrm{~nm}$, Whatman) and sealed with a fast curing medical grade silicone (Med2-4220, Nusil). The sensing platform and the electronic components were glued together using the USP class VI biocompatible glue (Loctite 3211 USP class VI biocompatible glue, purchased from Loctite). Component interconnection was realized with aluminum wire bonding and protected with a glob top. To further improve parylene $\mathrm{C}$ adhesion and moisture penetration, the assembled platform was treated with silane A-174 (from Merck) and coated with $16 \mu \mathrm{m}$ of parylene C (from Specialty Coating Systems), deposited by chemical vapor deposition using a Comelec C-30-S Parylene deposition system. The outer silicon shell was realized by placing the implant into a Plexiglas mold (realized by micromachining) and by injecting the biocompatible medical-grade silicone (Med-6033 from Nusil). To increase the host comfort, the outer shell was made $1 \mathrm{~mm}$ thick and with rounded corners.

In the second and third version of the packaging, the epoxy adhesive (EP42HT-2Med) system was used to assembly the electronic components and the sensing platform in the integrated device. The sensing platform was placed on the top of the PCB containing the ICs and the microprocessor. The interconnections between the pads of the sensing platform and the electronic components were realized with aluminum wire bonding and were protected with a glob top protection of 0.3 $\mathrm{mm}$. In the second version of the implantable device, all the edges of the device were rounded with a milling machine. Two subsequent $5 \mu \mathrm{m}$ layers of Parylene $\mathrm{C}$ were deposited by chemical vapor deposition using a Comelec C-30-S Parylene Deposition System. Parylene C was used to cover the whole device but not the electrode array that needs to be in contact with fluids. The electrodes were functionalized with MWCNTs

A

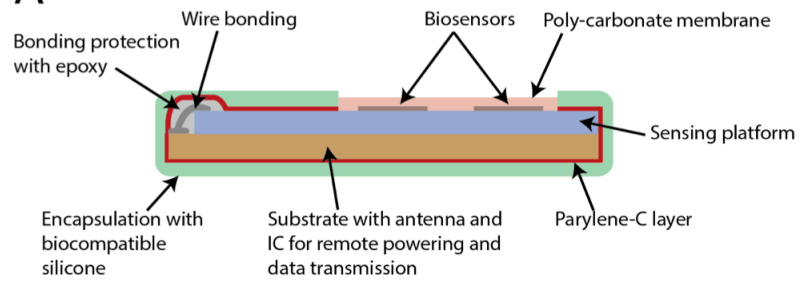

$(0.1 \mu \mathrm{l}$ of a chitosan/MWCNT suspension was then manually drop cast on the electrodes and dried in air). Finally the device was covered with three layers of a biocompatible membrane made by epoxy-enhanced polyurethane. For the biocompatible outer packaging, a homogeneous solution was obtained by mixing $125 \mathrm{mg}$ of an epoxy adhesive (EP42HT-2Med system), purchased by Master Bond (Hackensack, USA) as a certified biocompatible two-components adhesive, $112.5 \mathrm{mg}$ of polyurethane (Sigma Aldrich), $12.5 \mathrm{mg}$ of the surfactant agent polyethylene glycol ether (Brij ${ }^{\circledR 30}$, Sigma Aldrich), for $10 \mathrm{ml}$ of tetrahydrofuran (THF, Sigma Aldrich) used as solvent. Subsequent depositions of the membrane by dip coating were applied at $1 \mathrm{~h}$ intervals and then the sensors were stored overnight at room temperature. A fast curing at high temperature $\left(2 \mathrm{~h}\right.$ at $\left.80^{\circ} \mathrm{C}\right)$ is needed to ensure the biocompatibility of the resin. After this process the sensors were again kept overnight at room temperature, and then stored in PBS one day for membrane swelling

In the third version of the implantable device, for the electrode functionalization with MWCNTs, $0.1 \mu \mathrm{l}$ of a chitosan/MWCNT suspension was then manually drop cast on the electrodes and dried in air. The edges of the device were not rounded with a milling machine, and two different biocompatible silicone elastomers were used to cover and round the edges and the bottom of the device: a first layer was realized with the Kwik-cast sealant (from World Precision Instruments), and the second more external layer with a certified biocompatible elastomer silicone (Nusil MED2 4220). The sensing part was only covered with the epoxy-enhanced polyurethane membrane. The overall weight and volume of the implantable device are $0.61 \mathrm{~g}$ and $0.4 \mathrm{~cm}^{3}$, respectively.

Fig. 2 reports the scheme with the cross-sections of the three different implantable devices with the respective packaging.

\section{In-vivo biocompatibility tests, first packaging}

C57Bl/6 male mice (2 months old) were used for the experiments. The animals were bred and treated in accordance with the Swiss Federal Veterinary Office guidelines and were

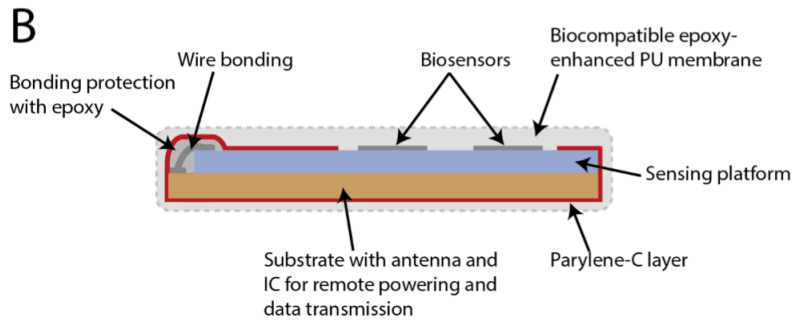

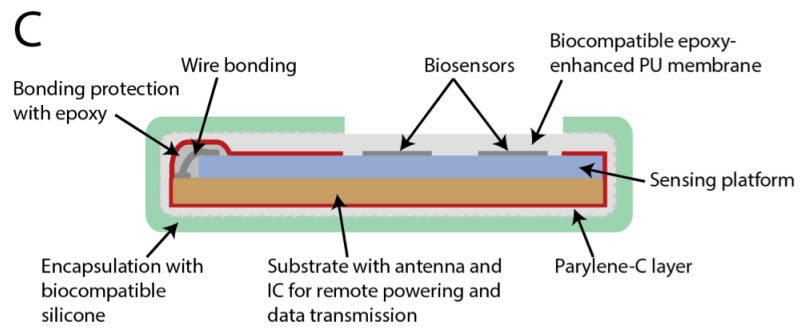

Fig. 2. Schematics of the three implantable devices: the first (A), the second (B), and the third (C). 
kept in specific pathogen-free animal facility. Experiments were approved by "Dipartimento della Sanità e Socialità" with authorization numbers TI-19/2010. Microchips were cleaned, disinfected with ethanol $70 \%$ and placed in sterile PBS (GIBCO). An Air Pouch (AP) was created by subcutaneous injection of sterile air in the back of the mice at day $1(5 \mathrm{~mL})$ and day $3(3 \mathrm{~mL})$; this procedure creates a cavity of $1.5 \mathrm{~cm}$ diameter and $0.5 \mathrm{~cm}$ height. At day 6 , mice were anesthetized with isoflurane $4 \%$, shaved and locally sterilized with Betadine Solution; the sterile microchips were implanted and the cavity sutured with Vicryl 6.0 (Provet AG). As a control of local inflammation, bacterial lipopolysaccharide (LPS, 50 $\mathrm{g}$ /mouse, LabForce AG) was injected daily into the cavity for the last 2 weeks of the 30 days of the experiment. As negative control APs were generated in the absence of any surgical procedure. After 30 days, the microchips were removed, the cavity was rinsed with $0.5 \mathrm{~mL}$ of PBS (Gibco) and the liquid collected and centrifuged at $7000 \mathrm{rpm}$ for $10^{\prime}$ at $4{ }^{\circ} \mathrm{C}$.

\section{In-vivo biocompatibility tests, second packaging}

For biocompatibility tests, the animals were bred and treated in accordance with the Swiss Federal Veterinary Office guidelines and were kept in specific pathogen-free animal facility. Experiments were approved by 'Dipartimento della Sanitá e Socialitá" with authorization number TI-19/2010. Microchips were cleaned and disinfected with ethanol 70\% and placed in sterile PBS (Gibco). An AP was created by subcutaneous injection of sterile air in the back of male C57BL/6 mice at day $1(5 \mathrm{~mL})$ and day $3(3 \mathrm{~mL})$ : this procedure creates a cavity of $1.5 \mathrm{~cm}$ diameter and $0.5 \mathrm{~cm}$ height. At day 6, mice were anesthetized with isoflurane $4 \%$, shaved and locally sterilized with Betadine solution; the sterile microchips were implanted and the cavity sutured with Vicryl 6.0 (Provet AG). As a control of local inflammation, LPS (50 $\mu \mathrm{g} /$ mouse, LabForce AG) was injected daily into the cavity for the last 2 weeks of the 30 days of the experiment. As negative control APs were generated in the absence of any surgical procedure. As further control commercial chips (DATA MARS) were injected through sterile needle. After 30 days, the microchips were removed, the cavity was rinsed with 0.5 $\mathrm{mL}$ of PBS (Gibco) and the liquid collected and centrifuged at $7000 \mathrm{rpm}$ for $10 \mathrm{~min}$ at $4{ }^{\circ} \mathrm{C}$.

\section{E. In-vivo biocompatibility tests, third packaging}

C57Bl/6 male mice (2 months old) were used for the experiments. The animals were bred and treated in accordance with the Swiss Federal Veterinary Office guidelines and were kept in specific pathogen-free animal facility. Experiments were approved by "Dipartimento della Sanità e Socialità" with authorization number TI-09/2013. Microchips were cleaned, disinfected with ethanol $70 \%$ and placed in sterile PBS (Gibco). Mice were anesthetized with isoflurane 4\%, shaved and locally disinfected with Betadine solution; the sterile microchips were implanted in the peritoneum and the cavity sutured with Vicryl 6.0 (Provet AG). After 30 days, the microchips were removed, the peritoneal cavity was rinsed with $0.5 \mathrm{~mL}$ of PBS (Gibco) and the liquid collected and centrifuged at $7000 \mathrm{rpm}$ for $10^{\prime}$ at $4{ }^{\circ} \mathrm{C}$. As negative control peritoneal wash was performed to mice with absence of any surgical procedure.

\section{F. In-vivo biocompatibility tests: assessment of the level of inflammation}

The concentration of ATP was determined in the supernatant with an ATP determination kit (Invitrogen). For polymorphonuclear neutrophils detection, the pellet was resuspended in $0.2 \mathrm{~mL}$ RPMI 10\% Fetal Bovine Serum (Gibco) and analyzed at flow cytometer (FACS Canto, Becton Dickinson) with antibodies specific for CD11b and Gr1, labeled with allophycocyanin (APC) and fluorescein isothiocyanate (FITC, BioLegend), respectively.

\section{RESULTS AND DISCUSSION}

The main features of an implantable device that can be sources of inflammation are the shape, the materials, and sizes of the device [10]. In this paper we compare three different packaging realized with different shape, size and with different materials. However, they all share some common features: (i) MWCNTs were entrapped in a chitosan matrix to prevent the potential toxicity due to their nano-particle nature; (ii) to prevent the corrosion of electronic components due to the contact with biologic fluids, and to prevent the leaking of potential hazardous substances, a coating of Parylene $\mathrm{C}$ was deposited [8,9]; (iii) a biocompatible packaging was realized, as, for an implantable sensor, it is essential for a correct wound healing, and for ensuring prolonged sensor functionality. The main differences between the three systems are: (i) the shape of the device; (ii) the shape of the packaging in silicone; (iii) the composition of the membrane that covers the sensors; (iv) the composition of the external packaging. All these differences are illustrated in Fig. 2.

To compare the properties of the different designs, the invivo biocompatibility of the implantable devices was investigated by subcutaneously implanting the prototypes in mice for 30 days. Moreover, in order to test if the position of the implant influences the final biocompatibility, the first two prototypes were implanted in the back of mice, and the third one was implanted in the peritoneum. At the end of the period, the implant site was washed with PBS, and levels of ATP and neutrophils in the elution liquid were quantified to follow the local inflammatory response, as ATP and neutrophils are both inflammatory mediators but they give different information.

Data from ATP and neutrophils for the first prototype, depicted in Fig. 3, suggest that after 30 days the host seems to accept the insert. Comparison with the commercial chip, level of neutrophil, and biocompatibility tests with cell cultures for the first version of the packaging have been published in [8].

Fig. 4 reports ATP and neutrophil variations in the liquid collected from the implant site for the second version of the packaging. ATP and neutrophil levels were also evaluated for a commercial chip (DATA MARS), for a negative control (mice with AP), and in case of artificial inflammation introduced by the injection of bacterial lipopolysaccharides (LPS).

As shown in the last column in Fig. 4, the negative control presents a barely detectable concentration of neutrophils, while there is still a small amount of extracellular ATP due to mechanical stress created during the collection of the fluid. Data from both ATP and neutrophil concentration suggest that the membrane alone provides already a quite good biocompatible coverage. 


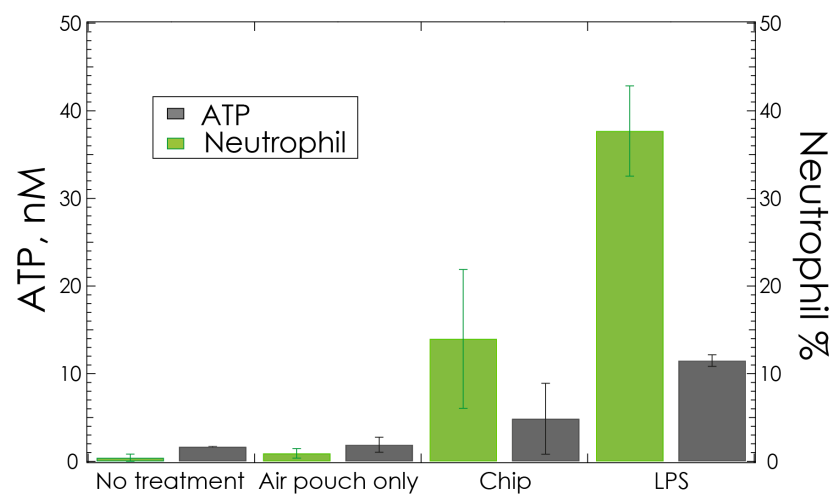

Fig. 3. ATP concentrations (nM) recovered from APs after 30 days from the implant of the first version of the implantable device.

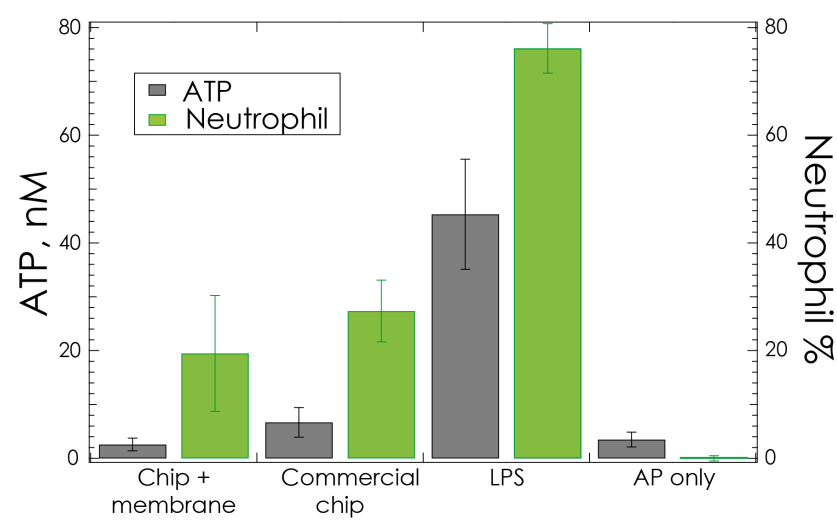

Fig. 4. ATP concentrations (nM) and \% of neutrophils recovered from APs after 30 days from the implant of the second version of the implantable device.

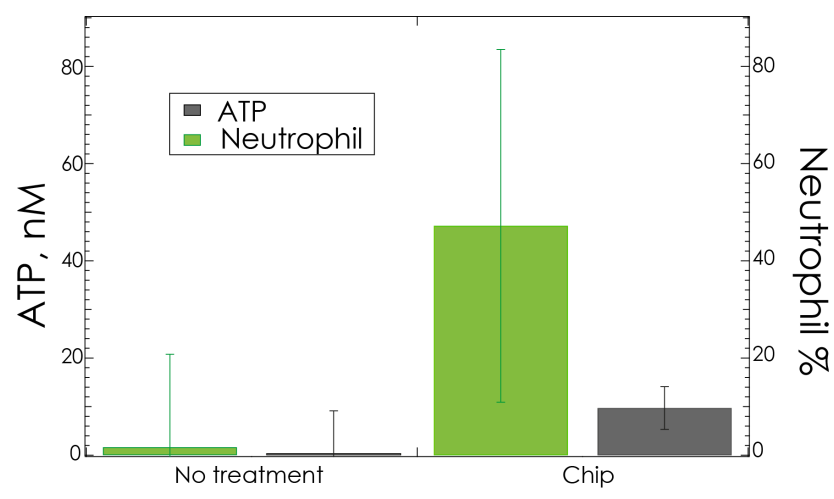

Fig. 5. ATP concentrations (nM) and \% of neutrophils recovered from APs after 30 days from the implant of the third version of the implantable device.

Furthermore, after 30 days, ATP and neutrophil levels are comparable with the negative control (AP), as well as for the commercial chip, and significantly lower than the positive control (LPS), proving that after 30 days the host seems to accept the implant.

We also performed in-vivo biocompatibility tests with the third version of the packaging, made by the epoxy enhanced polyurethane membrane and a biocompatible silicone, by implanting for 30 days four prototypes in the peritoneum of mice. The values for ATP and neutrophil concentration are reported in Fig. 5. Unfortunately, the level of both ATP and neutrophils was still high after 30 days compared to the negative control, proving that the host does not tolerate this implant, and that the surgery into the peritoneal cavity creates extensive cell damages.

\section{CONCLUSIONS}

From these tests we can conclude that the peritoneum is probably not the best place to implant the device, as the mouse can easily scratch the suture and increase the level of the inflammation. Moreover, data from the tests run with the first and second prototypes proved that both the PU-based membrane and the packaging made in silicone elastomer provide a quite good biocompatible coverage.

The membrane in polyurethane represents the best solution as a membrane for the sensing part, as the deposition technique is more reliable and reproducible than the procedure used to fix the polycarbonate membrane.

\section{REFERENCES}

[1] Carrara S., Ghoreishizadeh S.S., Olivo J., Taurino I., Baj-Rossi C., Cavallini A., de Beeck M.O., Dehollain C., Burleson W., Moussy F.G., Guiseppi-Elie A., De Micheli G., Fully Integrated Biochip Platforms for Advanced Healthcare, Sensors 2012, 12, 11013-11060

[2] Carrara S. (Aut), Bio/CMOS Interfaces and Co-Design, Springer, NY, 2013, ISBN 978-1-4614-4689-7

[3] Burleson W., Carrara S. (Ed): Security and Privacy for Implantable Medical Devices, Springer, NY, 2014, ISBN 978-1-4614-1673-9

[4] Olivo J., Carrara S., De Micheli G.. "Energy harvesting and remote powering for implantable biosensors." IEEE Sensors Journal 11 (2011): 1573-1586

[5] Kilinc, E. G., Kapucu, K., Maloberti, F., \& Dehollain, C. (2014, October). Servo-controlled remote powering and low-power data communication of implantable bio-systems for freely moving animals. In Biomedical Circuits and Systems Conference (BioCAS), 2014, 508-511

[6] de Beeck, M. O., Qian, K., Fiorini, P., Malachowski, K., \& Van Hoof, C. (2012). Design and characterization of a biocompatible packaging concept for implantable electronic devices. Journal of microelectronics and electronic packaging, 9(1), 43-50.

[7] Cavallini A., Baj-Rossi C., Ghoreishizadeh S.S., De Micheli G., Carrara S., "Design, fabrication, and test of a sensor array for perspective biosensing in chronic pathologies," in IEEE BioCAS 2012, Hsinchu, Taiwan, 2012, pp. 124-127.

[8] Cavallini, A.; Jost, T.R.; Ghoreishizadeh, S.S.; Olivo, J.; de Beeck, M.O.; Gorissen, B.; Grassi, F.; De Micheli, G.; Carrara, S., "A Subcutaneous Biochip for Remote Monitoring of Human Metabolism: Packaging and Biocompatibility Assessment," Sensors Journal, IEEE, vol.15, no.1, pp.417,424, Jan. 2015

[9] Baj-Rossi, C.; Kilinc, E.G.; Ghoreishizadeh, S.S.; Casarino, D.; Jost, T.R.; Dehollain, C.; Grassi, F.; Pastorino, L.; De Micheli, G.; Carrara, S., "Full Fabrication and Packaging of an Implantable Multi-Panel Device for Monitoring of Metabolites in Small Animals," Biomedical Circuits and Systems, IEEE Transactions on , vol.8, no.5, pp.636,647, Oct. 2014

[10] S. P. Nichols, A. Koh, W. L. Storm, J. H. Shin, and M. H. Schoenfisch, "Biocompatible materials for continuous glucose monitoring devices," Chemical reviews, vol. 113, no. 4, pp. 25282549,2013 